\title{
Renal function in HIV-infected children and adolescents treated with tenofovir disoproxil fumarate and protease inhibitors
}

\author{
Giuseppe Pontrelli', Nicola Cotugno*, Donato Amodio, Paola Zangari, Hyppolite K Tchidjou, Stefania Baldassari, \\ Paolo Palma and Stefania Bernardi
}

\begin{abstract}
Background: Kidney disease is an important complication in HIV infected people, and this may be related to infection or antiretroviral therapy (ART). Our aim is to assess renal function in HIV infected paediatric patients, who may be particularly affected and are likely to take ART for longer than adults, and investigate the long term role of Tenofovir Disoproxil Fumarate (TDF) alone or co-administered with Ritonavir-boosted Protease Inhibitors (PI).

Methods: Serum creatinine, phosphate and potassium levels, with estimated Glomerular Filtration Rate (eGFR), had been prospectively evaluated for 2 years in a cohort of HIV infected children and adolescents (age 9-18) on ART, and data analyzed according to the exposure to TDF or simultaneous TDF and PI.

Results: Forty-nine patients were studied (57\% female, mean age 14). Sixty-three percent were treated with ART containing TDF (Group A), and 37\% without TDF (Group B); 47\% with concomitant use of TDF and PI (Group C) and 53\% without this combination (Group D). The groups didn't differ for age, gender or ethnicity. The median creatinine increased in the entire cohort and in all the groups analyzed; eGFR decreased from $143.6 \mathrm{~mL} / \mathrm{min} / 1.73$ $\mathrm{m}^{2}$ at baseline to 128.9 after 2 years $(p=0.006)$ in the entire cohort. Three patients presented a mild eGFR reduction, all were on TDF+PI. Phosphatemia decreased significantly in the entire cohort $(p=0.0003)$ and in TDF + PI group $(p=0.0128)$ after 2 years. Five patients $(10 \%)$ developed hypophosphatemia (Division of Acquired Immune Deficiency AE grade 1 or 2), and four of them were on TDF+PI.
\end{abstract}

Conclusions: Renal function decrease and hypophosphatemia occur over time in HIV infected children and adolescents on ART. The association with co-administration of TDF and PI appears weak, and further studies are warranted.

\section{Background}

Antiretroviral therapy (ART) has reduced morbidity and mortality in HIV infected children as in adults [1,2], but not much is known on the effect of long-term exposure to ART in children, because registration studies have a limited observation time and are often not focused on adverse events related to drug interactions [3]. Kidney disease is an important complication in HIV infected people, and associated to increased risk of morbidity and mortality, related to viral infection (as in HIV associated nephropathy, HIVAN), or to ART [4].

\footnotetext{
* Correspondence: giuseppe.pontrelli@opbg.net; cotugnonicola@yahoo.it
University Department of Pediatrics. Ospedale Pediatrico Bambino Gesù,

* Correspondence: giuseppe.pontrelli@opbg.net; cotugnonicola@yahoo.it Piazza Sant'Onofrio, 4, 00165 Rome, Italy
}

(c) 2011 Pontrelli et al; licensee BioMed Central Ltd. This is an Open Access article distributed under the terms of the Creative Commons Attribution License (http://creativecommons.org/licenses/by/2.0), which permits unrestricted use, distribution, and reproduction in any medium, provided the original work is properly cited.
Tenofovir Disoproxil Fumarate (TDF) is a Nucleotide Reverse Transcriptase Inhibitor, which is considered a component of recommended regimens in major Clinical Guidelines for adults, for its efficacy, activity against hepatitis B, and availability in co-formulation allowing a single pill a day regimen [5]. TDF has a good safety profile but several studies highlighted its association with renal damage in adults, with cases of severe tubular dysfunction characterized by elevated creatinine, hypophosphataemia, hypokalemia, and more rarely Fanconi syndrome which may persist after withdrawal of the drug [6-12].

TDF is not licensed in Europe in patients less than 18 years of age but, by 2010, it received Food and Drug 
Administration (FDA) approval for use in patients aged 12-18 years and weighing more than $35 \mathrm{~kg}$. Nevertheless TDF has been already used as salvage therapy in paediatric patients for several years, often in association with boosted Protease Inhibitors (PI), and few reports described the use of this drug in children. Two studies determined a favourable safety profile for this drug $[13,14]$ whereas renal toxicity and bone density loss were reported by other studies both in adults and children [15-18].

Renal toxicity seems related to the mechanism of clearance of TDF which involves a glomerular filtration and a tubular phase of active secretion. During the tubular phase, Organic Anion Transporters (OAT1 and OAT3) are involved in the intracellular accessing of TDF, on the other hand, Multidrug Resistance associated Protein (MRP2 and MRP4) regulate the extracellular clearance of the drug [19]. Ritonavir and all PI, could have a significant role as cofactor in the pathogenesis of TDF-induced kidney damage. TDF clearance is slower in subjects receiving PI [20], with an increase of TDF-AUC ranging from 22 to $37 \%$ [21] and an accumulation of toxic drug levels in tubular cells [22]. The results of in vitro studies also suggest that protease inhibitors may inhibit the function of the MRP2 and MRP4 membrane transporters, and therefore increase the tubular concentration of TDF, and thus mitochondrial toxicity [23].

Our aim is to evaluate renal function in HIV infected paediatric patients on antiretroviral therapy (ART) and for the first time in a controlled study, the role of TDF and the concomitant use of TDF and PI.

\section{Methods}

A prospective observational study was conducted among a cohort of HIV infected children and adolescents $(<18$ years of age) on ART followed between 2008 and 2010 at Bambino Gesù Children Hospital of Rome, Italy. The study was approved by the local Ethical Committee, and informed consent obtained from the parents or guardians of all participants. The aim of the study was to investigate the role of TDF and concomitant use of TDF and PI on renal function in HIV infected children and adolescents. The cohort was therefore analyzed firstly using two groups according to the exposure to TDF (Group A: TDF) or not (Group B: no TDF). Secondly a further analysis was performed according to the exposure to concomitant use of TDF and PI (Group C: TDF $+\mathrm{PI}$ ) or not (Group D: no TDF+PI). Since TDF was used only in drug experienced children older than nine, we studied only patients above this age, in order to not introduce any age-related bias. Data on gender, age, ethnicity, CD4 T cell count and HIV-RNA (with a value of $<50$ copies $/ \mathrm{mL}$ defined as undetectable) were collected at baseline. Renal function was assessed at baseline, and after 1 and 2 years by evaluation of serum creatinine (assessed with non-enzymatic CREA2 method, a modified version of Jaffé reaction), phosphate, and potassium, and filtrate was estimated by eGFR (estimated Glomerular Filtration Rate) calculated with the Schwartz formula $(\mathrm{k}$ *height $(\mathrm{cm}) /$ creatinine $(\mathrm{mg} / \mathrm{dl})$; with $\mathrm{k}=0.55$, or 0.70 in males over the age of 13 years). No change in the analytical methods to measure serum creatinine or other markers took place during the study period. Reduction of renal function was classified according to the guidelines of the National Kidney Foundation: "mild" 60-89; "moderate" 30-59; "severe" 15-29; "renal failure" < 15 $\mathrm{ml} / \mathrm{min}$ per $1.73 \mathrm{~m}^{2}$. Hypophosphatemia was divided into four grades according to Division of Acquired Immune Deficiency (DAIDS) scale. For patients under 14 years old: "mild": 3.0-3.5; "moderate" 2.5-2.9; "severe" 1.5-2.4; "potentially life-threatening" $<1.5 \mathrm{mg} / \mathrm{dL}$. For patients older than 14: "mild": 2.5-3.0; "moderate" 2.02.4; "severe" 1-1.9; "potentially life-threatening" less than $1.0 \mathrm{mg} / \mathrm{dL}$.

Data for continuous variables were expressed as mean and standard deviation (SD) if normally distributed, or median and Inter-Quartile Range (IQR) if not normally distributed. Categorical data were showed as counts and percentages. Analysis was conducted using Epi Info version 3.5.1 (Centers for Diseases Control, Atlanta) or GraphPad Prism version 5 for Windows (GraphPad Software, San Diego California USA). Statistical analysis of baseline variables was carried out for continuous data with either ANOVA method for Analysis of Variance for normal distributed data, or Mann-Whitney test for non-normal distributed data, whereas chi-squared or Fisher exact tests were used for categorical data. Analyses of variables of renal function were performed with non-parametric Friedman test for comparison of values of baseline, first and second year in each group, with Wilcoxon matched-paired signed rank test for comparison of two time points, and with Mann-Whitney for analysis of values of Group A versus Group B, and values of Group C versus Group D at each time point. A two tailed P value of less than 0.05 was considered significant in all the analysis.

We expected an enrollment of about fifty patients, and about two-thirds exposed to TDF, and half to TDF + PI. Therefore, for eGFR analysis, the power to detect a difference of $15 \mathrm{~mL} / \mathrm{min} / 1.73 \mathrm{~m} 2$, considering a $95 \%$ Confidence Interval, and assuming a Standard Deviation of 20 , was $96 \%$ in the whole cohort, $86 \%$ in Group A, 59\% in the group B; $76 \%$ in the groups $\mathrm{C}$ and $\mathrm{D}$. The power to detect a difference of $15 \mathrm{~mL} / \mathrm{min} / 1.73 \mathrm{~m} 2$ between Group A and B is $71 \%$, and $76 \%$ between Group C and D. Regarding phosphatemia, the power to detect a difference of $0.5 \mathrm{mg} / \mathrm{dL}$ considering a $95 \%$ Confidence 
Interval, and assuming a Standard Deviation of 0.7, was $95 \%$ in the whole cohort, $83 \%$ in the Group A, $55 \%$ in the group $\mathrm{B} ; 71 \%$ in groups $\mathrm{C}$ and $\mathrm{D}$. The power to detect a difference of $0.5 \mathrm{mg} / \mathrm{dL}$ between Group A and B was $67 \%$, and $71 \%$ between Group C and D.

\section{Results}

Forty-nine patients (9-18 year old) were studied. They had a mean age of 13.6 years, and 28 (57\%) were female. The mean CD $4 \%$ value was 27.1 ( \pm 10.2$)$, corresponding to value of 565 cells $/ \mu L ; 47 \%$ had HIV-RNA undetectable, and $39 \%$ of patients had a history of CDC (Center for Disease Control) class $C$ clinical diagnosis, and no patients had creatinine $>1 \mathrm{mg} / \mathrm{dL}$ at baseline. None of the patients was treatment naive.

Sixty-three percent were treated with antiretroviral (ARV) regimens containing Tenofovir (Group A: TDF), and 37\% without TDF (Group B: No TDF).

Forty-seven percent were treated with regimens containing both TDF and PI (Group C: TDF+PI), and 53\% without this combination (Group D: No TDF+PI). Specifically, $16 \%$ of patients were on TDF without PI, 20\% were with PI but alternative NRTIs, and $16 \%$ with other regimens containing neither TDF nor PI. The children didn't differ for age, gender, frequency of undetectable HIV-RNA, or ethnicity ( $p>0.05$ for all comparisons) both between Group A and Group B, and between Group C and D. CD4 resulted lower in group A and Group C if compared to Group B and D respectively (Table 1).

As shown in Figure 1, in the entire cohort there was a significant increase of serum creatinine, from a median of $0.62 \mathrm{mg} / \mathrm{dL}$ at baseline to $0.73 \mathrm{mg} / \mathrm{dL}(p<0.0001)$ after 2 years; eGFR decrease from a median of 143.6 at baseline to 135.6 at 1 year, and $128.9 \mathrm{ml} / \mathrm{min}$ per 1.73 $\mathrm{m}^{2}$ at 2 years $(p=0.006)$. The value of phosphatemia decreased significantly from 4.4 at baseline to 4.0 at 1 year $(p=0.0301)$ and to $3.8 \mathrm{mg} / \mathrm{dl}$ at 2 years $(p=$ $0.0003)$. The median of potassiemia in the entire cohort during the study period didn't vary significantly.

The creatinine increased significantly after 2 years in each of the groups analyzed, from +0.10 in Group A and $\mathrm{C}$ to $+0.16 \mathrm{mg} / \mathrm{dL}$ in Group B. There were no significant differences between Group A and B, and Group $\mathrm{C}$ and $\mathrm{D}$.

The eGFR decreased after 2 years in all groups, significantly in Group B $\left(-23.8 \mathrm{ml} / \mathrm{min}\right.$ per $\left.1.73 \mathrm{~m}^{2}, p=0.012\right)$ and Group D $(-19.1, p=0.02)$. There were no significant differences between Group A and B, and Group C and D. Among the subgroups we observed that 7 patients on TDF+PI, 2 on PI and not in TDF, 1 in TDF and not PI, and 2 not taking nor TDF nor PI showed an increase in eGFR during the study period. On the other hand only 1 patient on TDF remained stable and all the others (37 patients) showed a decrease in eGFR. Three patients developed a mild reduction in glomerular filtration rate $\left(60-89 \mathrm{ml} / \mathrm{min}\right.$ per $\left.1.73 \mathrm{~m}^{2}\right)$, all were treated with both TDF and PI. Among these patients none received any other nephrotoxic drugs during the entire study period and none of them experienced infections or malignancy.

The phosphatemia decreased after 2 years in each group, significantly in Group A $(-0.5 \mathrm{mg} / \mathrm{dL}, p=0.0058)$ and Group $C(-0.5, p=0.0128)$. There were no significant differences between Group A and B, and Group C and D. Five patients developed a grade 1 or 2 DAIDS hypophosphatemia event; among these, 4 were treated with TDF+PI and one only with TDF. Phosphorus levels came back to normal without discontinuing the therapy. Among these patients two were $\mathrm{HCV}$ co infected, not taking any HCV treatment, and one patient experienced Burkitt Lymphoma and underwent chemotherapy.

\section{Discussion}

Our study describes for the first time in a controlled study, the role of TDF and the concomitant use of TDF and PI in HIV-infected paediatric patients. The long term impact of ARV, specifically of drugs associated with renal damage on adults, is a major topic in infected children, since they are expected to have a longer duration of exposure. Moreover registration studies of antiretrovirals in children are few, with limited time of observation, and are often not focused on drugs interaction.

We reported a significant variation in the eGFR after 2 years in the entire cohort $(p=0.006)$ with a mean net loss of $12.1 \mathrm{ml} / \mathrm{min}$ per $1.73 \mathrm{~m}^{2}$, confirming what was reported by other studies on HIV infected adults $[24,25]$. We didn't find any significant eGFR variation in patients taking TDF+PI or TDF, even when compared to the group that was not treated with nor TDF nor PI, or TDF respectively. However, all three of the cases who developed a mild reduction in eGFR were on a regimen containing both TDF and PI.

This is consistent with two other studies on ARVexperienced children treated with TDF-containing regimens which didn't find evidence of impaired filtration rate, even if their sample sizes were very small (27 and 26 children respectively) and were not controlled $[13,14]$, and with other pediatric cohort studies reporting some cases of renal toxicity associated with TDF [26-28]. Furthermore, as reported in other studies, estimated eGFR cannot be considered a sensitive indicator of early renal damage related to TDF [29], and our observation could have been affected by limited sample size. In fact, other studies on adults, with a large cohort and a higher power, found that patients treated with TDF and PI based regimens had a significantly greater 
Table 1 Baseline characteristics of the patients, overall and in each treatment group

\begin{tabular}{|c|c|c|c|c|c|c|c|}
\hline & \multirow{2}{*}{$\begin{array}{l}\text { Total } \\
\text { n.49 }\end{array}$} & \multicolumn{3}{|c|}{ Exposure to TDF } & \multicolumn{3}{|c|}{ Exposure to concomitant use of TDF and PI } \\
\hline & & $\begin{array}{c}\text { A } \\
\text { TDF } \\
\text { n.31 (63\%) }\end{array}$ & $\begin{array}{c}\text { B } \\
\text { NO TDF } \\
\text { n.18 (37\%) }\end{array}$ & $\mathrm{p}$ & $\begin{array}{c}\mathrm{C} \\
\text { TDF+PI } \\
\text { n.23 (47\%) }\end{array}$ & $\begin{array}{c}\text { D } \\
\text { NO TDF+PI } \\
\text { n.26 (53\%) }\end{array}$ & $\mathbf{p}$ \\
\hline Female $n(\%)$ & $28(57 \%)$ & $18(58 \%)$ & $10(56 \%)$ & 0.86 & $12(52 \%)$ & $16(62 \%)$ & 0.51 \\
\hline Age Mean (SD) & $13.6( \pm 2.5)$ & 14.0 & 13.0 & 0.18 & $14.1( \pm 2.7)$ & $13.2( \pm 2.3)$ & 0.20 \\
\hline HIV $<50$ copies $/ \mathrm{mL}$ & $23(47 \%)$ & $12(39 \%)$ & $11(61 \%)$ & 0.13 & $9(39 \%)$ & $14(54 \%)$ & 0.30 \\
\hline CD4\% (SD) & $27.1( \pm 10.2)$ & $24.7( \pm 10.4)$ & $31.8( \pm 8.3)$ & 0.04 & $23.6( \pm 10.5)$ & $30.7( \pm 8.8)$ & 0.02 \\
\hline Ethnicity (Africa) & $8(16 \%)$ & $3(10 \%)$ & $5(28 \%)$ & 0.10 & $2(9 \%)$ & $6(23 \%)$ & 0.17 \\
\hline $\begin{array}{l}\text { eGFR at baseline median } \\
\mathrm{ml} / \mathrm{min} \text { per } 1.73 \mathrm{~m}^{2}(\mathrm{IQR})\end{array}$ & $143.6(124.5-167.4$ & $143.6(123.7-165.4)$ & $147.5(124.5-169.6)$ & $0.48^{*}$ & $135.0(122.2-156.0)$ & $152.6(128.0-169.6)$ & $0.14^{*}$ \\
\hline $\begin{array}{l}\text { Phosphatemia at baseline } \\
\text { Median } \mathrm{mg} / \mathrm{dl} \text { (IQR) }\end{array}$ & $4.4(3.8-5.0)$ & $4.4(3.8-5.0)$ & $4.7(3.8-5.2)$ & $0.30^{*}$ & $4.4(3.9-5.0)$ & $4.5(3.8-5.1)$ & $0.44^{*}$ \\
\hline $\begin{array}{l}\text { Years on current } \\
\text { regimen mean (SD) }\end{array}$ & $1.11(1,64)$ & $0,76(1,30)$ & $1,71(1,99)$ & 0.06 & $1(1,41)$ & $1,21(1,84)$ & 0.67 \\
\hline \multicolumn{8}{|l|}{ ART regimen } \\
\hline $\mathrm{TDF}+\mathrm{FTC}+\mathrm{LPV} / \mathrm{r}$ & $10(20 \%)$ & $10(32 \%)$ & - & - & $10(43 \%)$ & & - \\
\hline $\mathrm{TDF}+\mathrm{FTC}+\mathrm{ATV} / \mathrm{r}$ & $11(22 \%)$ & $11(35 \%)$ & - & - & $11(48 \%)$ & & - \\
\hline TDF+FTC+Other $\mathrm{Pl} / \mathrm{r}$ & $2(4 \%)$ & $2(6 \%)$ & - & - & $2(9 \%)$ & & - \\
\hline$A B C+3 T C+L P V / r$ & $6(12 \%)$ & - & $6(33 \%)$ & - & & $6(23 \%)$ & - \\
\hline $\mathrm{ABC}+3 \mathrm{TC}+$ other $\mathrm{PI}$ & $4(8 \%)$ & - & $4(22 \%)$ & - & - & $4(15 \%)$ & - \\
\hline $3 \mathrm{TC}+\mathrm{ABC}+\mathrm{EFV}$ & $7(14 \%)$ & - & 7 (39\%) & - & - & $7(27 \%)$ & - \\
\hline $\mathrm{ABC}+3 \mathrm{TC}+\mathrm{AZT}$ & $1(2 \%)$ & - & $1(6 \%)$ & - & - & $1(4 \%)$ & - \\
\hline $\mathrm{TDF}+\mathrm{ABC}+\mathrm{EFV}$ & $1(2 \%)$ & $1(3 \%)$ & - & - & - & $1(4 \%)$ & - \\
\hline $\mathrm{TDF}+\mathrm{FTC}+\mathrm{EFV}$ & $7(14 \%)$ & $7(23 \%)$ & - & - & - & $7(27 \%)$ & - \\
\hline
\end{tabular}

SD Standard Deviation; $p$ value calculated with ANOVA or with Mann Whitney test $\left(^{*}\right)$ and was referred to comparison between baseline variable of Group A versus Group B, or Group C versus Group D. TDF Tenofovir Disoproxil Fumarate; FTC= Emtricitabine; LPV/r = Lopinavir/ritonavir; ATV/r Atazanavir/ritonavir; PI/r= Ritonavir boosted Protease Inhibitors; $A B C=$ Abacavir; $3 T C=$ Lamivudine; $E F V=$ Efavirenz; $A Z T=$ Zidovudine; OT Other Treatments; $r=$ ritonavir; $I Q R$ interquartile range

decline in renal function than those taking TDF with Non-NRTIs (NNRTIs) or non-TDF-based regimens [25,30]. The role of co-administration of TDF and PI on renal function was also recently demonstrated in a large randomized clinical trial on 1858 ART-naïve adults, the ACTG 5202 study, comparing four different regimens (TDF+NNRTI; TDF+PI; other NRTIs+NNRTI; otherNRTIs+PI). In this study the only regimen with a significant reduction in estimated creatinine clearance was in the group on both TDF and PI [31].

The detection of early or mild TDF-related nephrotoxicity could be evaluated with other more specific tests for proximal tubule injury, such as serum phosphate and potassium levels.

In our study we didn't find any significant variation in potassium in all therapy groups, but there was a trend in reduction in Group C (TDF+PI) after 2 years, and this variation resulted significantly different if compared to the Group D (no TDF+PI). This seems to confirm what has been already demonstrated by observations in adults and in pediatric population on TDF $[12,26]$.
In healthy population serum phosphorus is slightly higher in the prepubertal age, decreasing to adult values after puberty, as also considered by the age-specific DAIDS event scale. A recent case-control study in children confirmed an association between the use of TDF and hypophosphatemia, but it didn't analyze the role of concomitant use of PI [17]. In our analysis the levels of phosphatemia changed earlier (after 1 year) and significantly in the entire cohort. Moreover phosphate reduction resulted significant and worse in the group treated with TDF, and in the group treated with TDF and PI; notably four of the five patients who developed clinical hypophosphataemia were on this regimen.

The observation of a tubular damage associated with concomitant use of TDF and PI is consistent with pathogenetic cellular studies and PK analysis, showing that PI inhibition of MRP2 and MRP4 results in an increase of tubular concentration of TDF [22,23,32], and TDF clearance is slower in subjects receiving PI, with an AUC increased of $22-37 \%$, and possible increase of mitochondrial damage [21]. 

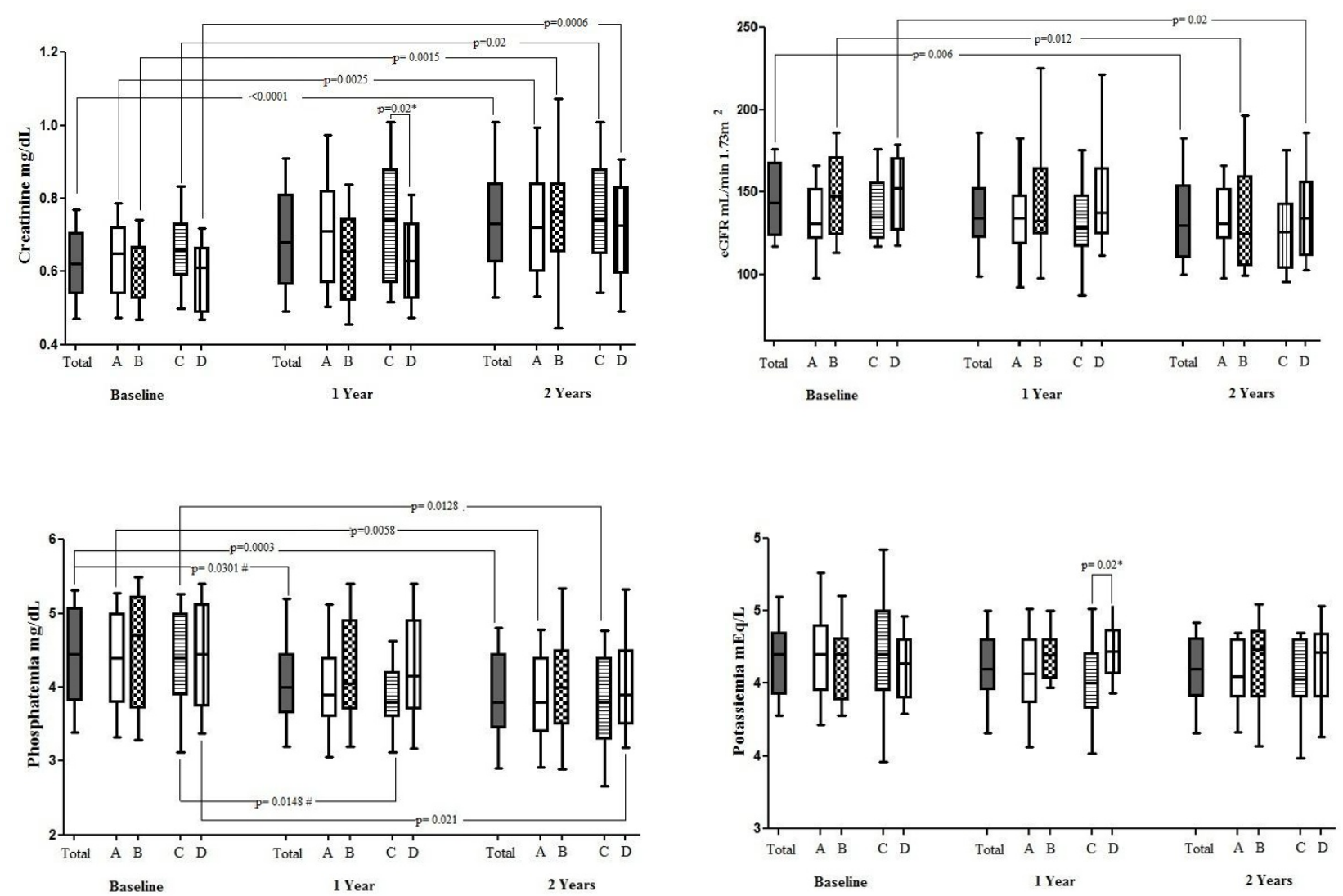

Figure 1 Renal function and tubular damage markers, at baseline and after 1 and 2 years, in the entire cohort, and in each treatment group. Patients were grouped according to the exposure to antiretrovirals: Group A = TDF, Group B = no TDF; Group C = TDF+PI, Group D = no TDF+PI. Statistical analysis related to baseline, 1 and 2 years in each group were performed through Friedman test. P-values related to analysis between group A versus group B and group $C$ versus group D at each time point were obtained through Mann Whitney test $\left(^{*}\right)$, while those related to analysis between two time points among the same group with Wilcoxon matched-paired signed rank test (\#).

However our analysis has some limitations: it is an observational study, and although the groups analyzed resulted not different for age, gender, HIV or ethnicity, other variables like immune status and disease progression, could have had a role. Furthermore we assessed renal impairment without analyzing urine tests and other more sensitive indicators such as $\beta-2$ microglobulinuria that may have shown some milder renal abnormalities. Moreover, we didn't assess the association of TDF and PI with lower bone mineral density as reported by other studies $[13,17,33]$, and the clinical significance of kidney abnormalities observed remains not fully known. Further studies with more sensitive urine markers, bone density assays, and longer follow-up are warranted.

\section{Conclusion}

In conclusion, this study highlights that in HIV infected children and adolescents on ART, renal function and phosphatemia decreased over time. Cases of hypophosphatemia and mild reduction of eGFR observed in patients on TDF+PI suggest an association between this ART regimen and renal function, however this appears weak and not confirmed in the subgroup analysis. New studies, with more sensitive methods and larger number, focused on the effect of these regimens on renal function of HIV infected children are warranted.

\section{Acknowledgements}

We would like to acknowledge all the patients who participated in this study. We want to acknowledge Miss Mariateresa Bernardi and Lisbeth Damas for the English review of the manuscript.

\section{Authors' contributions}

GP and SB designed the study; GP and NC wrote the paper and equally contributed to this work; DA, PZ, HKT, PP provided input to the data; GP and DA performed the statistical analysis. All authors contributed to the interpretation of the data, commented the manuscript, and approved the final version.

\section{Competing interests}

The authors declare that they have no competing interests.

Received: 30 June 2011 Accepted: 23 January 2012

Published: 23 January 2012 


\section{References}

1. D'Arminio Monforte A, Sabin CA, Phillips A, et al: The changing incidence of AIDS events in patients receiving highly active antiretroviral therapy. Arc Intern Med 2005, 165:416-423.

2. Chiappini E, Galli L, Tovo PA, et al: Changing patterns of clinical events in perinatally HIV-1-infected children during the era of HAART. AIDS 2007, 21:1607-1615.

3. Guideline on the clinical development of medical products for the treatment of HIV infection. [http://www.ema.europa.eu/docs/en_GB/ document_library/Scientific_guideline/2009/09/WC500003399.pdf]

4. Roling J, Schmid H, Fischereder M, et al: HIV-Associated Renal Diseases and Highly Active Antiretroviral Therapy-Induced Nephropathy. Clin Infect Dis 2006, 42:1488-1495.

5. Panel on Antiretroviral Guidelines for Adults and Adolescents. Guidelines for the use of antiretroviral agents in HIV-1-infected adults and adolescents. Department of Health and Human Services 2011, 1-166, Available at [http://www.aidsinfo.nih.gov/ContentFiles/ AdultandAdolescentGL.pdf].

6. Lee JC, Marosok RD: Acute tubular necrosis in a patient receiving tenofovir. AIDS 2003, 17:2543-2544.

7. Creput C, Gonzalez-Canali G, Hill G, et al: Renal lesions in HIV-1-positive patient treated with tenofovir. AIDS 2003, 17:935-937.

8. Barrios A, Garcia-Benayas T, Gonzalez-Lahoz J, et al: Tenofovir related nephrotoxicity in HIV-infected patients. AIDS 2004, 18:960-963.

9. Zimmermann AE, Pizzoferrato T, Bedford J, et al: Tenofovir-associated acute and chronic kidney disease: a case of multiple drug interactions. Clin Infect Dis 2006, 42:283-290.

10. Izzedine $H$, Launay-Vacher V, Isnard-Bagnis C, et al: Drug induced Fanconi's syndrome. Am J Kidney Dis 2003, 41:292-309.

11. Gupta SK: Tenofovir-associated Fanconi syndrome: review of the FDA adverse event reporting system. AIDS Patient Care STDS 2008, 22:99-103.

12. Cirino CM, Kan VL: Hypokalemia in HIV patients on tenofovir. AIDS 2006, 20(12):1671-1673.

13. Hazra R, Gafni Rl, Maldarelli F, et al: Tenofovir disoproxil fumarate and an optimized background regimen of antiretroviral agents as salvage therapy for pediatric HIV infection. Pediatrics 2005, 116(6):846-854

14. Viganò $A$, Bedogni $G$, Manfredini $V$, et al: Long-term renal safety of tenofovir disoproxil fumarate in vertically HIV-infected children adolescents and young adults: a 60-month follow-up study. Clin Drug Investig 2011, 31(6):407-415.

15. Hussain S, Khayat A, Tolaymat A: Nephrotoxicity in a child with perinatal HIV on tenofovir, didanosine and lopinavir/ritonavir. Pediatr Nephrol 2006, 21:1034-1036.

16. Rollot F, Nazal E, Chauvelot-Moachon L, et al: Tenofovir-related Fanconi syndrome with nephrogenic diabetes insipidus in a patient with acquired immunodeficiency syndrome: the role of lopinavirritonavirdidanosine. Clin Infect Dis 2003, 37:174-176.

17. Judd A, Boyd KL, Stöhr W, et al: Effect of tenofovir disoproxil fumarate on risk of renal abnormality in HIV-1-infected children on antiretroviral therapy: a nested case-control study. AIDS 2010, 24(4):525-534.

18. Purdy JB, Gafni RI, Reynolds JC, et al: Decreased bone mineral density with off-label use of tenofovir in children and adolescents infected with human immunodeficiency virus. J Pediatr 2008, 152:582-584.

19. Rodriguez-Novoa S, Labarga P, Soriano V: Pharmacogenetics of tenofovir treatment. Pharmacogenomics 2009, 10(10):1675-1685.

20. Kiser JJ, Carten ML, Aquilante $\mathrm{CL}$, et al: The effect of lopinavir/ritonavir on the renal clearance of tenofovir in HIV-infected patients. Clin Pharmacol Ther 2008, 83(2):265-272.

21. Viread: SUMMARY OF PRODUCT CHARACTERISTICS. European Medicines Agency [http://www.ema.europa.eu/docs/en_GB/document_library/ EPAR Product_Information/human/000419/WC500051737.pdf pag.8].

22. Kiser JJ, Fletcher CV, Flynn PM, et al: Pharmacokinetics of antiretroviral regimens containing tenofovir disoproxil fumarate and atazanavirritonavir in adolescents and young adults with human immunodeficiency virus infection. Adolescent Trials Network for HIV/ AIDS Interventions. Antimicrob Agents Chemother 2008, 52(2):631-637.

23. Robertson EE, Ranking GO: Human renal organic anion transporters: characteristics and contributors to drug and drug metabolite excretion. Pharmacol Ther 2006, 109:383-389.

24. Gallant JE, Moore RD: Renal function with use of a tenofovir-containing initial antiretroviral regimen. AIDS 2009, 23:197-1975.
25. Campbell $L$, Ibrahim F, Fisher $M$, et al: Spectrum of chronic kidney disease in HIV-infected patients. HIV Med 2009, 10(6):329-336.

26. Riordan A, Judd A, Boyd K, et al: Tenofovir use in Human immunodeficiency virus-1-infected children in the United Kingdom and Ireland. Pediatr Infect Dis J 2009, 28:204-209.

27. Hussain S, Khayat A, Tolaymat A, et al: Nephrotoxicity in a child with perinatal HIV on tenofovir, didanosine and lopinavir/ritonavir. Pediatr Nephrol 2006, 21(7):1034-1036.

28. Soler-Palacín P, Melendo S, Noguera-Julian A, et al: Prospective study of renal function in HIV-infected pediatric patients receiving tenofovircontaining HAART regimens. AIDS 2011, 25(2):171-176.

29. Labarga P, Barreiro P, Martin-Carbonero L, et al: Kidney tubular abnormalities in the absence of impaired glomerular function in HIV patients treated with tenofovir. AIDS 2009, 23(6):689-696.

30. Goicoechea M, Liu S, Best B, et al: Greater tenofovir-associated renal function decline with protease inhibitor-based versus nonnucleoside reverse-transcriptase inhibitor-based therapy. J Infect Dis 2008, 197:102-108.

31. Daar E, Tierney C, Fischl M, et al: ACTG 5202: final results of ABC/3TC or TDF/FTC with either EFV or ATV/r in treatment-naive HIVinfected patients. [Abstract 59LB.]. 17th Conference on Retroviruses and Opportunistic Infections San Francisco, CA; 2010.

32. Kiser JJ, Aquilante $\mathrm{CL}$, Anderson PL, et al: Effect of Multidrug resistance proteins 2 and 4 polymorphisms on tenofovir pharmacokinetics in HIVinfected patients. 7th International Workshop on Clinical Pharmacology of HIV Therapy Lisbon, Portugal; 2006.

33. Giacomet V, Mora S, Martelli L, et al: A 12-month treatment with tenofovir does not impair bone mineral accrual in HIV-infected children. J Acquir Immune Defic Syndr 2005, 40:448-450.

\section{Pre-publication history}

The pre-publication history for this paper can be accessed here: http://www.biomedcentral.com/1471-2334/12/18/prepub

doi:10.1186/1471-2334-12-18

Cite this article as: Pontrelli et al:: Renal function in HIV-infected children and adolescents treated with tenofovir disoproxil fumarate and protease inhibitors. BMC Infectious Diseases 2012 12:18.

\section{Submit your next manuscript to BioMed Central and take full advantage of:}

- Convenient online submission

- Thorough peer review

- No space constraints or color figure charges

- Immediate publication on acceptance

- Inclusion in PubMed, CAS, Scopus and Google Scholar

- Research which is freely available for redistribution

Submit your manuscript at www.biomedcentral.com/submit
C Biomed Central 